Volume 2, Issue 1, pages 67-74

p-ISSN 2655-8564, e-ISSN 2685-9432

\title{
Application of Klein-4 Group on Domino Card
}

\author{
Asido Saragih ${ }^{1, *}$, Santri Chintia Purba ${ }^{2}$ \\ ${ }^{1}$ Department of Mathematics, Satya Wacana Christian University, Salatiga, \\ Indonesia \\ ${ }^{2}$ Department of Educational Mathematics, Christian University \\ of Indonesia, Jakarta, Indonesia \\ *Corresponding Author: saragihasido@gmail.com
}

(Received 04-11-2019; Revised 03-02-2020; Accepted 03-02-2020)

\begin{abstract}
Klein-4 or Klein-V group is a group with four elements including identity. The binary operation on Klein-4 group will produce identitiy if operated to it self and produce another non-identity element if operated to another nonidentity element. The focus of this paper is to explain the principle of Klein4 group on Domino card and also find all complete possible elements. Domino card is a set of 28 cards which the surface of each card divided in two identic boxes contain combination of dots as pattern. The final part of this research is to find out all possible combination of cards which can be used as elements of a Klein-4 groups.
\end{abstract}

Keywords: klein, domino

\section{Introduction}

Group is a set of nonempty elements with a well define binary operation which satisfy conditions for binary operation are associative, has identity and inverse element, 


\section{International Journal of Applied Sciences and Smart Technologies}

Volume 2, Issue 1, pages 67-74

p-ISSN 2655-8564, e-ISSN 2685-9432

and close under binary operation [1]. We notice a group $G$ with binary operation $*$ as $(G, *)$.

\section{Definition 1.1.}

Let $(G, *)$ be a group. Then $G$ is an abelian group if elements of $G$ are commutative under $*$. The name of abelian group is actually was given for honors a Norwegian mathematics Niels Hendrik Abel [2] . Further for our convenience, let us denote $\{p\}^{k}$ be $k-t h$ repetitions of $p$ under the operation of binary operation *. For example $\{p\}^{2}=p * p$, and $\{p\}^{4}=p * p * p * p$.

Let $p$ and $q$ are two numbers such that $0 \leq p \leq 6$ and $0 \leq q \leq 6$. We construct an abreviation with equivalent meaning as $0 \leq p, q \leq 6$ to help us in simplification writing.

\section{Definition 1.2.}

Let $(G, *)$ be a group, and $p, q, e \in G$, with $e$ is the identity. We say $p$ is generator of $q$ if there exists a positive integer $n$ such that $\{p\}^{n}=q$. In this case it is not surprise to make an understending that $p$ generate $q$. Furthermore, we say a positive integer $m$ be the order of $p$ if $\{p\}^{m}=e$.

\section{Definition 1.3.}

Let $(G, *)$ be a group. Then we say $G$ is a cyclic group if there exists an element $p \in G$ and $p$ generate all elements of $G[3]$.

Basically there are a lot of group we can construct. But there is one simple and interesting group named Klein-4 group which also oftenly signed as $V_{4}$ stand for "Vier", means "four" in German and "Klein" means "small" [4]. A Klein-4 group or Klein-V group is a group with four elements including identity. Klein-4 with a binary operation - and $e$ as identity, will produce $e$ if an element operated to it self and produce it self if operated with $e$. If two different non-identity elements are operated by $\bullet$, will produce the another non-identity element. For example, let $(G, \bullet)=\{e, a, b, c\}$ 


\section{International Journal of Applied Sciences and Smart Technologies}

Volume 2, Issue 1, pages 67-74

p-ISSN 2655-8564, e-ISSN 2685-9432

as a Klein-4 group. Then, the result of the operation over - represented on Table 1 below :

Table 1. Klein-4 group

\begin{tabular}{|l|l|l|l|l|}
\hline$\bullet$ & $e$ & $a$ & $b$ & $c$ \\
\hline$e$ & $e$ & $a$ & $b$ & $c$ \\
\hline$a$ & $a$ & $e$ & $c$ & $b$ \\
\hline$b$ & $b$ & $c$ & $e$ & $a$ \\
\hline$c$ & $c$ & $b$ & $a$ & $e$ \\
\hline
\end{tabular}

Certainly, the simplicity concept of Klein-4 group makes it become interesting to observe. Collecting four object with a binary operation to represent Klein-4 group would not be easy to define. However, group theorists tried to find Klein-4 group applications in daily life. In order to reach that purpose, of course they need to find the generator elements to generate it. For example, Samuel and Mshelia [5] found one of the its application on a game called "Tsorry Checkerboard" which consists of $2 \times 2$ boxes with some rule and moving code. On another side Rietman, Robert and Jack [6] explain about Klein-4 group in genetic coding. Especially for its identity concept, Danckwerts and Neubert [7] mention it on their paper regarding the symmetries of genetic code-doublets.

Theorem 1.1.

Let $(G, \bullet)$ be a Klein-4 group with e as identity. Then the cyclic subgroup of $(G, \bullet)$ is only contain identity e and another element such that maximum order of cyclic subgroup of $(G, \bullet)$ is 2 .

Proof. Let $\mathrm{p} \in \mathrm{G}$. If $p=e$, then $\{p\}$ is a trivial cyclic subgroup of $G$. If $p \neq e$, then $p \bullet p=e$. Hence, for any $n, m \in \mathbb{Z}$ we have $p \bullet p=\left(p^{2}\right)^{n}=e^{n}=e$. Let $m$ be an odd number. Then we always have $n$ such that $m=2 n+1$. This fact allowed us to have $p=p^{m}$, since $p^{m}=p^{2 n+1}=p^{2 n} * p=e * p=p$. Therefore, $p$ is the generator of $\{p\}$ and $\{e, p\}$. Then we have prove our assertion. 


\section{International Journal of Applied Sciences and Smart Technologies}

Volume 2, Issue 1, pages 67-74

p-ISSN 2655-8564, e-ISSN 2685-9432

Domino cards is a set of 28 cards with every card's surface devided in to two boxes and contain the combination of 0 up to 6 dots. We can represent the combination of dots on the card's surfaces as paired of positive integer number as :

$$
\begin{aligned}
& (0,0),(0,1),(0,2),(0,3),(0,4),(0,5),(0,6), \\
& (1,1),(1,2),(1,3),(1,4),(1,5),(1,6), \\
& (2,2),(2,3),(2,4),(2,5),(2,6), \\
& (3,3),(3,4),(3,5),(3,6), \\
& (4,4),(4,5),(4,6), \\
& (5,5),(5,6), \\
& (6,6) .
\end{aligned}
$$

We also need to remind that for domino card, $(p, q)=(q, p)$ where $0 \leq p, q \leq 6$. Hence there are no two or more than two cards in a set of domino cards consists the same number of dots on their surfces. We can look for this example in Figure 1 below :

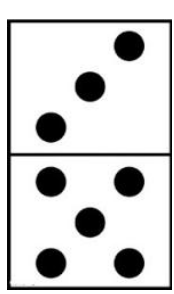

$(3,5)$

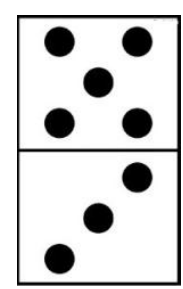

$(5,3)$

Figure 1. Domino card

Further, in the next section we will discuss about the principle of Klein-4 groups which applied to domino cards as our main discuss section.

\section{Klein-4 Group Principle On Domino Card}

Suppose we have a Klein-4 group $(G, \bullet)$ with elements are four different domino cards. Let us define the binary operation $\bullet$ : deleting the same pattern on card. This binary operation will implies $\bullet$ only be able to operate between two cards which have at least one side the same pattern. For example

$$
\begin{aligned}
& (6,1) \cdot(0,6)=(0,1) \\
& (3,0) \cdot(0,2)=(2,3) \\
& (6,6) \cdot(6,6)=(0,0)
\end{aligned}
$$




\section{International Journal of Applied Sciences and Smart Technologies}

Volume 2, Issue 1, pages 67-74

p-ISSN 2655-8564, e-ISSN 2685-9432

Following Klein-4 group definition, we need to have four different cards as elements including the identity.

Theorem 2.1.

Let $(G, \bullet)$ be a Klein-4 group with $\bullet$ define as "deleting the same pattern on card". Then $(0,0)$ is the identity.

Proof. Let $(p, q)$ be an arbitrary card element of $(G, \bullet)$ such that $0 \leq p, q \leq 6$. Since we need to have a card which operated by $\bullet$ to it self produces identity, then $(p, q) \bullet(p, q)$ will implies $p$ deleting $p$ and $q$ deleting $q$, hence we have $(p, q) \bullet(p, q)=(0,0)$. Then we obtain our assertion.

\section{Remark 2.2.}

Remaining three elements of Klein-4 group are defined as the combination of $(p, q) \cdot(q, r)=(p, r)$, where $0 \leq p, q, r \leq 6$.

As we stated $(0,0)$ to be our identity, here we attach all combinations of possible elements of Klein-4 group on Domino card with binary operation $\bullet$. We can see all the combination in Table 2 below :

Table 2. Combinations of all possible elements

\begin{tabular}{|c|c|c|c|}
\hline$e$ & $a$ & $b$ & $c$ \\
\hline \multirow{15}{*}{$(0,0)$} & \multirow{5}{*}{$(0,1)$} & $(1,2)$ & $(0,2)$ \\
\hline & & $(1,3)$ & $(0,3)$ \\
\hline & & $(1,4)$ & $(0,4)$ \\
\hline & & $(1,5)$ & $(0,5)$ \\
\hline & & $(1,6)$ & $(0,6)$ \\
\hline & \multirow{4}{*}{$(0,2)$} & $(2,3)$ & $(0,3)$ \\
\hline & & $(2,4)$ & $(0,4)$ \\
\hline & & $(2,5)$ & $(0,5)$ \\
\hline & & $(2,6)$ & $(0,6)$ \\
\hline & \multirow{3}{*}{$(0,3)$} & $(3,4)$ & $(0,4)$ \\
\hline & & $(3,5)$ & $(0,5)$ \\
\hline & & $(3,6)$ & $(0,6)$ \\
\hline & \multirow{2}{*}{$(0,4)$} & $(4,5)$ & $(0,5)$ \\
\hline & & $(4,6)$ & $(0,6)$ \\
\hline & $(0,5)$ & $(5,6)$ & $(0,6)$ \\
\hline
\end{tabular}




\section{International Journal of Applied Sciences and Smart Technologies}

Volume 2, Issue 1, pages 67-74

p-ISSN 2655-8564, e-ISSN 2685-9432

\section{Theorem 2.3.}

Let $(p, q)$ be a non-identity element of $(G, \bullet)$. Maximum order of $(p, q)$ is 2 .

Proof. Since $(p, q)$ is a non-identity of $(G, \bullet)$, then value of both of $p$ and $q$ are between 0 and 6 where $p=0$ and $q=0$ will not exists on the same card. This condition leads us to $(p, q)^{1}=(p, q)$ and $(p, q)^{2}=(p, q) \bullet(p, q)=e$. Then we obtain our assertion.

\section{Conclusion}

Klein 4 group is a simple group consists of four elements including one of them as identity. Many researchers found its application in real life over many field outside of mathematics. This paper presented one more its application by using domino cards as elements by choosing one well define binary operation and many important supporting theorem. Finally the porpose of this paper has been achieved.

\section{References}

[1] M. Eie and S. T. Chang, A Course on Abstract Algebra, World Scientific, Singapore, 2009.

[2] W. K. Nicholson, Introduction to Abstract Algebra Fourth Edition. Wiley, USA, 2012.

[3] J. A. Gallian, Contemporary Abstract Algebra Eighth Edition, BROOKS/COLE CEN- GAGE Learning, USA, 2016.

[4] J. Minac and S. Chebolu, "Representations of the miraculous klein group", Ramanujan Mathematics Society Newsletter, 22 (1), 135 - 145, 2012.

[5] S. H. Tsok and I. B. Mshelia, "Application of Group Theory to a Local Game Called "Tsorry Checkerboard" (A Case of Klein Four- Group)", IOSR Journal of Mathematics, 7 (3), 4-6, 2013. 


\section{International Journal of Applied Sciences and Smart Technologies}

Volume 2, Issue 1, pages 67-74

p-ISSN 2655-8564, e-ISSN 2685-9432

[6] E. A. Rietman, R. L. Karp and J. A. Tuszynski, " Review and application of group theory to molecular systems biology ", Theoretical Biology and Medichal Modeling, 8 (21),1 - 29, (2011).

[7] H. J. Danckwerts and D. Neubert, "Symmetries of genetic code-doublets", Journal of Molecular Evolution 5, 327 - 332,(1975). 
International Journal of Applied Sciences and Smart Technologies

Volume 2, Issue 1, pages 67-74

p-ISSN 2655-8564, e-ISSN 2685-9432

This page intentionally left blank 